\title{
Green and Efficient Microwave Assisted Synthesis of Schiff Bases and Hydroxyl Derivatives of 1, 3, 4-Thiadiazole Containing $N$-Methyl Piperazine Moiety and their Antimicrobial and Antioxidant Potential
}

\author{
CHANDRA PRAKASH GHARU \\ Department of Chemistry, Government College Merta City, Nagaur, Rajasthan, India \\ chandrapushp@gmail.com
}

Received 23 June 2014 / Accepted 26 June 2014

\begin{abstract}
A new class of thiadiazole having combination of Schiff base and Mannich base containing $N$-methyl piperazine moiety was synthesized by an efficient microwave assisted green synthetic approach. Antioxidant activity of methanolic solutions of synthesized compounds was determined by Reducing power assay and Hydrogen peroxide scavenging activity at $700 \mathrm{~nm}$ and $250 \mathrm{~nm}$ respectively. The synthesized compounds were also screened for antbacterial activity and were characterized by FTIR, ${ }^{1} \mathrm{H}$ NMR and elemental analysis.
\end{abstract}

Keywords: Thiadiazole, Schiff bases, Mannich bases, $N$-methyl piperazine, Antimicrobial activity, Antioxidant activity

\section{Introduction}

Microwave assisted synthesis have emerged as an efficient approach owing to enhanced rates, higher selectivity, efficiency and higher yield. 1,3,4-Thiadiazole are widely exposed to therapeutic world because of their known antimicrobial ${ }^{1-3}$, antitubercular ${ }^{4}$, anti-helicobacter pylori activity ${ }^{5}$. Piperazine and its derivatives have their own importance in drug discovery and have inhibited the growth of certain microbes ${ }^{6-8}$. Free radicals are reactive oxygen species (ROS) which are generated during oxidation of food in our body to gain energy. They are short lived, unstable, highly reactive and capable of attacking a healthy cell of the body, causing them to lose their structure and function. Antioxidants are capable of stabilizing or deactivating a free radical before they attack the healthy cell. Some synthesized heterocyclic compounds are reported to show remarkable antioxidant activity ${ }^{9-12}$. Toxicity and resistance play an important role in drug development for treating diseases caused by microbes.

Thus there is a need of new class of compounds having antimicrobial and antioxidant potential. In view of above findings thiadiazole and piperazine moieties are incorporated together to give confined structure like the title compounds for evaluating its antimicrobial and antioxidant activities. 


\section{Experimental}

Melting points were determined in open capillary tubes in a 'Innco' electrical apparatus and are uncorrected. FTIR was carried out on Schimadzu 8101 A. Spectrophotometer in $\mathrm{KBr}$ pellets and ${ }^{1} \mathrm{HNMR}$ was recorded on a DPX $300 \mathrm{MHz}$ Brucker Spectrophotometer in DMSO with chemical shift in ppm. MW irradiations were carried out in domestic Samsung microwave oven, model number 310 EMENO 22332. The synthesized products (Scheme 1) were frequently checked by thin layer chromatography (TLC). Absorbance for antioxidant activity was determined by ELICO SL 177 scanning mini spec.

\section{Synthesis of 2-nitro-4-N-methyl piperazino aniline (Ia)}

Equimolar ratio of $N$-methyl piperazine and $p$-chloro, o-nitroaniline in benzene was irradiated in a microwave oven for 15 minutes. After the completion of the reaction, the mixture was left to cool to room temperature. The resultant solid was filtered, dried and recrystallized from ethanol to yield the pure product. The purity of compound was checked by running TLC

Yield: $70 \%$, M.P. $80{ }^{0}$ C; M.W. 236, M.F. $\mathrm{C}_{11} \mathrm{H}_{16} \mathrm{~N}_{4} \mathrm{O}_{2}$, Calcd. (Found), N \%=4.21 (4.28); FTIR (KBr, cm $\left.{ }^{-1}\right): 3340(\mathrm{NH}), 1530\left(\mathrm{NO}_{2}\right)$, PMR (DMSO, $\delta$ ppm, $\left.300 \mathrm{MHz}\right): 2.16$ (s, 3H, - $\left.\mathrm{CH}_{3}\right), 2.65$ (br, 4H, $\left.\mathrm{CH}_{3}-\mathrm{N}\left(\mathrm{CH}_{2}\right)_{2}\right), 2.74$ (br, 4H, $\left.\mathrm{Ar}-\mathrm{N}\left(\mathrm{CH}_{2}\right)_{2}\right), 7.45-7.78(\mathrm{~m}, 3 \mathrm{H}$. $\mathrm{ArH}), 9.18$ (s, 2H, $\mathrm{NH}_{2}$ )

\section{Synthesis of 2-nitro-4-N-methyl piperazino aniline thiosemicarbazide (Ib)}

0.01 mole of 2-nitro 4- $N$-methyl piperazino aniline was dissolved in $20 \mathrm{~mL}$ of ammonia solution followed by gradual addition of 0.01 mole $\mathrm{CS}_{2} .20 \mathrm{~mL}$ of ethanol was added and stirring was continued till all the $\mathrm{CS}_{2}$ was dissolved. In an another beaker sodium salt of mono chloro acetic acid was prepared by dissolving equimolar ratio of sodium hydroxide and mono chloro acetic acid in minimum quantity of water to yield sodium salt of mono chloro acetic acid. The solution of the salt was then added to the reaction mixture followed by gradual addition of $10 \mathrm{~mL}$ hydrazine hydrate with continuous stirring. The mixture became warm which was cooled. On cooling, a solid separated out which was filtered, dried and recrystallized from ethanol.

Yield 65\%, M.P.-90 ${ }^{0}$ C, M.W. 310, M. F. $\mathrm{C}_{12} \mathrm{H}_{18} \mathrm{~N}_{6} \mathrm{O}_{2} \mathrm{~S}$, Calcd. (Found), N \%=3.69 (3.58), FTIR (KBr, cm $\left.{ }^{-1}\right): 3360(\mathrm{NH}), 1540\left(\mathrm{NO}_{2}\right), 1280(\mathrm{C}=\mathrm{S})$, PMR (DMSO, $\delta \mathrm{ppm}, 300$ $\mathrm{MHz}$ ): 2.12 (s, 3H, $\left.-\mathrm{CH}_{3}\right), 2.60$ (br, 4H, $\left.\mathrm{CH}_{3}-\mathrm{N}\left(\mathrm{CH}_{2}\right)_{2}\right), 2.70$ (br, $\left.4 \mathrm{H}, \mathrm{Ar}-\mathrm{N}\left(\mathrm{CH}_{2}\right)_{2}\right), 5.20$ 5.60 (br, 3H, NH-NH 2$), 7.45-7.78$ (m, 3H. ArH), 9.18 (s, 1H, NH)

\section{Synthesis of 5-(2'-nitro, 4'-N-methyl-piperazino anilino)-2-mercapto-1,3,4-thiadiazole} (Ic)

0.01 mole of the above prepared thiosemicarbazide and carbon disulphide $(0.01$ mole) were taken in a flask and irradiated in microwave oven in presence of DMF $(5 \mathrm{~mL})$ for 15 minutes. The solution was cooled and poured into ice cold water. The solid which separated out was filtered, dried and recrystallized from ethanol.

Yield: $75 \%$, M.P. $120{ }^{0}$ C, M.W. 352, M.F. $\mathrm{C}_{13} \mathrm{H}_{16} \mathrm{~N}_{6} \mathrm{O}_{2} \mathrm{~S}_{2}$, Calcd. (Found), N $\%=4.19(4.20)$, FTIR $\left(\mathrm{KBr}, \mathrm{cm}^{-1}\right): 3360(\mathrm{NH}), 3220(\mathrm{NH}), 1540\left(\mathrm{NO}_{2}\right), 1280(\mathrm{C}=\mathrm{S})$, $1610(-\mathrm{C}=\mathrm{N})$, PMR (DMSO, $\delta \mathrm{ppm}, 300 \mathrm{MHz}): 2.15\left(\mathrm{~s}, 3 \mathrm{H},-\mathrm{CH}_{3}\right), 2.62\left(\mathrm{br}, 4 \mathrm{H}, \mathrm{CH}_{3}-\right.$ $\left.\mathrm{N}\left(\mathrm{CH}_{2}\right)_{2}\right), 2.74$ (br, 4H, Ar- $\left.\left(\mathrm{CH}_{2}\right)_{2}\right), 5.20(\mathrm{~s}, 1 \mathrm{H}, \mathrm{NH}-\mathrm{C}=\mathrm{S}), 7.40-7.60$ (m, 3H. ArH), $9.10(\mathrm{~s}, 1 \mathrm{H}, \mathrm{NH})$. 
Synthesis of 5-(2'-nitro, 4'-N-methyl-piperazino anilino)-2-mercapto-3-p-anisidino/ morpholino/piperidino methyl-1,3,4-thiadiazole (Id, Ie, If)

Equimolar ratio of 5-(2'-nitro, 4'- $N$-methyl-piperazino-anilino)-2-mercapto-1,3,4thiadiazole, $p$-anisidine and formaldehyde was irradiated in ethanol for 15 minutes. Solid obtained on cooling was filtered, washed with water, dried and recrystallized by ethanol to yield the compound Id. Two other Mannich bases were synthesized by similar manner.

5-(2'-Nitro, 4'-N-methyl-piperazino anilino)-2-mercapto-3-p-anisidino methyl-1,3,4thiadiazole (Id)

Yield: 80\%, M.P. 202-204 ${ }^{0}$ C, M.W. 487, M.F. $\mathrm{C}_{21} \mathrm{H}_{25} \mathrm{~N}_{7} \mathrm{O}_{3} \mathrm{~S}_{2}$, Calcd. (Found), $\mathrm{N} \%=4.96$ (4.98), FTIR (KBr, cm $\left.{ }^{-1}\right): 3362(\mathrm{NH}), 1640(-\mathrm{C}=\mathrm{N}), 1535\left(\mathrm{NO}_{2}\right), 1282(\mathrm{C}=\mathrm{S})$, PMR (DMSO, $\delta$ ppm, $300 \mathrm{MHz}$ ): $2.12\left(\mathrm{~s}, 3 \mathrm{H},-\mathrm{CH}_{3}\right), 2.65$ (br, 4H, $\left.\mathrm{CH}_{3}-\mathrm{N}\left(\mathrm{CH}_{2}\right)_{2}\right), 2.72$ (br, 4H, $\left.\operatorname{Ar}-\mathrm{N}\left(\mathrm{CH}_{2}\right)_{2}\right), 3.20\left(\mathrm{~d}, 2 \mathrm{H}, \mathrm{CH}_{2}\right), 4.12\left(\mathrm{~s}, 3 \mathrm{H}, \mathrm{OCH}_{3}\right), 7.42-7.60(\mathrm{~m}, 7 \mathrm{H} . \mathrm{ArH}), 8.20(\mathrm{t}, 1 \mathrm{H}$, $\mathrm{NH}), 9.10$ (s, 1H, NH).

5-(2'-Nitro, 4'-N-methyl-piperazino anilino)-2-mercapto-3- morpholino methyl-1,3,4thiadiazole (Ie)

Yield: $78 \%$, M.P. $198-200{ }^{0}$ C, M.W. 451, M.F. $\mathrm{C}_{18} \mathrm{H}_{25} \mathrm{~N}_{7} \mathrm{O}_{3} \mathrm{~S}_{2}$, Calcd. (Found), N \%=4.60 (4.64), FTIR (KBr, cm $\left.{ }^{-1}\right): 3370(\mathrm{NH}), 1650(-\mathrm{C}=\mathrm{N}) 1530\left(\mathrm{NO}_{2}\right), 1275(\mathrm{C}=\mathrm{S})$, PMR (DMSO, $\delta \mathrm{ppm}, 300 \mathrm{MHz}): 2.14\left(\mathrm{~s}, 3 \mathrm{H},-\mathrm{CH}_{3}\right), 2.66$ (br, 4H, $\left.\mathrm{CH}_{3}-\mathrm{N}\left(\mathrm{CH}_{2}\right)_{2}\right), 2.70$ (br, 4H, Ar$\left.\mathrm{N}\left(\mathrm{CH}_{2}\right)_{2}\right), 3.20\left(\mathrm{~s}, 2 \mathrm{H}, \mathrm{CH}_{2}\right), 3.69-3.72$ (br, 8H, N-( $\left.\left.\mathrm{CH}_{2}\right)_{4}-\mathrm{O}\right)$ 7.42-7.60 (m, 3H. ArH), 9.10 (s, 1H, NH)

5-(2'-Nitro, 4'-N-methyl-piperazino anilino)-2-mercapto-3- piperidino methyl1,3,4-thiadiazole (If)

Yield:76\%, M.P. 195-196 ${ }^{0}$ C, M.W. 449, M.F. $\mathrm{C}_{19} \mathrm{H}_{27} \mathrm{~N}_{7} \mathrm{O}_{2} \mathrm{~S}_{2}$, Calcd. (Found), N \%=4.58 (4.60), FTIR (KBr, cm $\left.{ }^{-1}\right): 3362(\mathrm{NH}), 1645(-\mathrm{C}=\mathrm{N}), 1540\left(\mathrm{NO}_{2}\right), 1270(\mathrm{C}=\mathrm{S})$, PMR (DMSO, $\delta$ ppm, $300 \mathrm{MHz}): 2.10\left(\mathrm{~s}, 3 \mathrm{H},-\mathrm{CH}_{3}\right), 2.54-\left(\mathrm{br}, 10 \mathrm{H}, \mathrm{N}-\left(\mathrm{CH}_{2}\right)_{5}\right), 2.64$ (br, 4H, $\left.\mathrm{CH}_{3}-\mathrm{N}\left(\mathrm{CH}_{2}\right)_{2}\right), 2.70$ (br, $\left.4 \mathrm{H}, \mathrm{Ar}-\mathrm{N}\left(\mathrm{CH}_{2}\right)_{2}\right), 3.25\left(\mathrm{~s}, 2 \mathrm{H}, \mathrm{CH}_{2}\right), 7.50-7.58$ (m, 3H. ArH), 9.12 (s, $1 \mathrm{H}, \mathrm{NH})$,

Synthesis of 5-(2'-nitro, 4'-N-methyl-piperazino anilino)-2-mercapto-3-p-anisidino methylene-4' '-hydroxy-3' '-methoxy-bezilidene-1,3,4-thiadiazole (Id $)$

A mixture of 4-hydroxy,3-methoxy benzaldehyde (0.01 mole), Mannich base 5-(2'-nitro, 4'$N$-methyl-piperazino anilino)-2-mercapto-3-p-anisidino methyl-1,3,4-thiadiazole (Id) (0.01 mole) and anhydrous sodium acetate $(0.02$ mole) was irradiated in acetic acid for 15 mins. After cooling solution was poured in to ice cold water and kept overnight. The resulting solid was filtered, washed with hot water, dried and recrystallized from ethanol.

Other Schiff bases were synthesized in similar manner described above.

5-(2'-Nitro, 4'-N-methyl-piperazino anilino)-2-mercapto-3-p-anisidino methylene4',-hydroxy -3' 'methoxy benzilidene-1,3,4-thiadiazole (Id $\left.{ }_{a}\right)$

Yield: $74 \%$, M.P. $192-195{ }^{0}$ C, M.W. 621 , M.F. $\mathrm{C}_{29} \mathrm{H}_{31} \mathrm{~N}_{7} \mathrm{O}_{5} \mathrm{~S}_{2}$, Calcd. (Found), $\mathrm{N} \%=6.34$ (6.36), FTIR (KBr, cm $\left.{ }^{-1}\right): 3580(\mathrm{OH}), 3362(\mathrm{NH}), 3240(\mathrm{NH}), 1535\left(\mathrm{NO}_{2}\right), 1640(-\mathrm{C}=\mathrm{N})$, $1282(\mathrm{C}=\mathrm{S})$, PMR (DMSO, $\delta \mathrm{ppm}, 300 \mathrm{MHz}): 2.12\left(\mathrm{~s}, 3 \mathrm{H},-\mathrm{CH}_{3}\right), 2.63$ (br, 4H, $\mathrm{CH}_{3}$ $\left.\mathrm{N}\left(\mathrm{CH}_{2}\right)_{2}\right), 2.70\left(\mathrm{br}, 4 \mathrm{H}, \mathrm{Ar}-\mathrm{N}\left(\mathrm{CH}_{2}\right)_{2}\right), 3.70(\mathrm{~s}, 1 \mathrm{H},=\mathrm{CH}), 4.12\left(\mathrm{~s}, 3 \mathrm{H}, \mathrm{OCH}_{3}\right), 7.40-7.70(\mathrm{~m}$, 10H. ArH), 8.20 (s, 1H, NH), $8.70(\mathrm{~s}, 1 \mathrm{H}, \mathrm{NH}), 9.10(\mathrm{~s}, 1 \mathrm{H}, \mathrm{NH}), 10.10(\mathrm{~s}, 1 \mathrm{H}, \mathrm{OH})$. 
5-(2'-Nitro, 4'-N-methyl-piperazino anilino)-2-mercapto-3-p-anisidino methylene4'-hydroxy methyl benzilidene-1,3,4-thiadiazole ( $\left.\mathbf{I d}_{\boldsymbol{b}}\right)$

Yield: 72\%, M.P. 196-198 ${ }^{0}$ C, M.W. 605, M.F.C ${ }_{29} \mathrm{H}_{31} \mathrm{~N}_{7} \mathrm{O}_{4} \mathrm{~S}_{2}$, Calcd. (Found), N \%=6.17 (6.15), FTIR (KBr, cm $\left.{ }^{-1}\right): 3370(\mathrm{NH}), 1540\left(\mathrm{NO}_{2}\right), 1275(\mathrm{C}=\mathrm{S}), 1638(-\mathrm{C}=\mathrm{N}), 3250(\mathrm{NH})$, $3570(\mathrm{OH}), \mathrm{PMR}(\mathrm{DMSO}, \delta \mathrm{ppm}, 300 \mathrm{MHz}): 2.10\left(\mathrm{~s}, 3 \mathrm{H},-\mathrm{CH}_{3}\right), 2.20\left(\mathrm{~s}, 3 \mathrm{H},-\mathrm{CH}_{3}\right), 2.66$ (br, $\left.4 \mathrm{H}, \mathrm{CH}_{3}-\mathrm{N}\left(\mathrm{CH}_{2}\right)_{2}\right), 2.74$ (br, $\left.4 \mathrm{H}, \mathrm{Ar}-\mathrm{N}\left(\mathrm{CH}_{2}\right)_{2}\right), 4.08\left(\mathrm{~s}, 3 \mathrm{H}, \mathrm{OCH}_{3}\right), 7.45-7.66(\mathrm{~m}, 11 \mathrm{H}$. $\mathrm{ArH}), 8.18$ (s, 1H, NH), 9.10 (s, 1H, NH), $10.12(\mathrm{~s}, 1 \mathrm{H}, \mathrm{OH})$

5-(2'-Nitro, 4'-N-methyl-piperazino anilino)-2-mercapto-3- morpholino methylene4''-hydroxy 3' 'methoxy benzilidene-1,3,4-thiadiazole (I $\left.\boldsymbol{I}_{\mathbf{a}}\right)$

Yield: 78\%, M.P. 189-192 ${ }^{0}$ C, M.W. 585, M.F. $\mathrm{C}_{26} \mathrm{H}_{31} \mathrm{~N}_{7} \mathrm{O}_{5} \mathrm{~S}_{2}$, Calcd. (Found), N \%=5.96 (5.99), FTIR $\left(\mathrm{KBr}, \mathrm{cm}^{-1}\right): 3580(\mathrm{OH}), 3370(\mathrm{NH}), 1525\left(\mathrm{NO}_{2}\right), 1640(-\mathrm{C}=\mathrm{N}), 1280(\mathrm{C}=\mathrm{S})$, 1250 (C-O-C), PMR (DMSO, $\delta$ ppm, $300 \mathrm{MHz}$ ): 2.18 (s, 3H, $\left.-\mathrm{CH}_{3}\right), 2.65$ (br, 4H, $\mathrm{CH}_{3}-$ $\left.\mathrm{N}\left(\mathrm{CH}_{2}\right)_{2}\right), 2.72$ (br, $\left.4 \mathrm{H}, \mathrm{Ar}-\mathrm{N}\left(\mathrm{CH}_{2}\right)_{2}\right), 3.70-3.74$ (br, 8H, N- $\left.\left(\mathrm{CH}_{2}\right)_{4}-\mathrm{O}\right), 3.80(\mathrm{~s}, 1 \mathrm{H},=\mathrm{CH})$, $4.02\left(\mathrm{~s}, 3 \mathrm{H}, \mathrm{OCH}_{3}\right), 7.24-7.29(\mathrm{~m}, 6 \mathrm{H} . \mathrm{ArH}), 9.18(\mathrm{~s}, 1 \mathrm{H}, \mathrm{NH}), 10.12(\mathrm{~s}, 1 \mathrm{H}, \mathrm{OH})$

5-(2'-Nitro, 4'-N-methyl-piperazino anilino)-2-mercapto-3- morpholino methylene4'’-hydroxy methyl benzilidene-1,3,4-thiadiazole $\left(\mathbf{I e}_{\boldsymbol{b}}\right)$

Yield: $74 \%$, M.P. $180-182{ }^{0}$ C, M.W. 569 , M.F. $\mathrm{C}_{26} \mathrm{H}_{31} \mathrm{~N}_{7} \mathrm{O}_{4} \mathrm{~S}_{2}$, Calcd. (Found), N \%=5.81 (5.84), FTIR (KBr, cm $\left.{ }^{-1}\right): 3590(\mathrm{OH}), 3382(\mathrm{NH}), 1644(-\mathrm{C}=\mathrm{N}), 1535\left(\mathrm{NO}_{2}\right), 1282(\mathrm{C}=\mathrm{S})$, 1250 (C-O-C), PMR (DMSO, $\delta \mathrm{ppm}, 300 \mathrm{MHz}): 2.12\left(\mathrm{~s}, 3 \mathrm{H},-\mathrm{CH}_{3}\right), 2.22\left(\mathrm{~s}, 3 \mathrm{H},-\mathrm{CH}_{3}\right)$, 2.60 (br, $\left.4 \mathrm{H}, \mathrm{CH}_{3}-\mathrm{N}\left(\mathrm{CH}_{2}\right)_{2}\right), 2.74$ (br, $\left.4 \mathrm{H}, \mathrm{Ar}-\mathrm{N}\left(\mathrm{CH}_{2}\right)_{2}\right), 3.72-3.75$ (br, 8H, N- $\left.\left(\mathrm{CH}_{2}\right)_{4}-\mathrm{O}\right)$, 7.20-7.25 (m, 7H. ArH), 9.08 (s, 1H, NH), 10.08 (s, 1H, OH)

5-(2'-Nitro, 4'-N-methyl-piperazino anilino)-2-mercapto-3- piperidino methylene4',-hydroxy -3' 'methoxy- benzilidene-1,3,4-thiadiazole (If $\left.\boldsymbol{f}_{a}\right)$

Yield: $80 \%$, M.P. $190-194{ }^{0}$ C, M.W. 583, M.F. $\mathrm{C}_{27} \mathrm{H}_{33} \mathrm{~N}_{7} \mathrm{O}_{4} \mathrm{~S}_{2}$, Calcd. (Found), $\mathrm{N} \%=5.95$ (5.98), FTIR (KBr, cm $\left.{ }^{-1}\right): 3580(\mathrm{OH}), 3364(\mathrm{NH}), 1640(-\mathrm{C}=\mathrm{N}), 1533\left(\mathrm{NO}_{2}\right), 1274(\mathrm{C}=\mathrm{S})$, PMR (DMSO, $\delta$ ppm, $300 \mathrm{MHz}): 2.12\left(\mathrm{~s}, 3 \mathrm{H},-\mathrm{CH}_{3}\right), 2.50-\left(\mathrm{br}, 10 \mathrm{H}, \mathrm{N}-\left(\mathrm{CH}_{2}\right)_{5}\right), 2.62$ (br, $\left.4 \mathrm{H}, \mathrm{CH}_{3}-\mathrm{N}\left(\mathrm{CH}_{2}\right)_{2}\right), 2.72\left(\mathrm{br}, 4 \mathrm{H}, \mathrm{Ar}-\mathrm{N}\left(\mathrm{CH}_{2}\right)_{2}\right), 3.72(\mathrm{~s}, 1 \mathrm{H},=\mathrm{CH}), 4.02\left(\mathrm{~s}, 3 \mathrm{H}, \mathrm{OCH}_{3}\right), 7.30-$ 7.38 (m, 6H. ArH), 9.10 (s, 1H, NH), $10.12(\mathrm{~s}, 1 \mathrm{H}, \mathrm{OH})$

5-(2'-Nitro, 4'-N-methyl-piperazino anilino)-2-mercapto-3- piperidino methylene4'-hydroxy methyl benzilidene-1,3,4-thiadiazole (If $\left.\boldsymbol{f}_{\boldsymbol{b}}\right)$

Yield: $82 \%$, M.P. $198-200{ }^{0}$ C, M.W. 567, M.F. $\mathrm{C}_{27} \mathrm{H}_{33} \mathrm{~N}_{7} \mathrm{O}_{3} \mathrm{~S}_{2}$, Calcd. (Found), N \%=5.77 (5.78), FTIR $\left(\mathrm{KBr}, \mathrm{cm}^{-1}\right): 3580(\mathrm{OH}), 3380(\mathrm{NH}), 1640(-\mathrm{C}=\mathrm{N}), 1530\left(\mathrm{NO}_{2}\right), 1270(\mathrm{C}=\mathrm{S})$, PMR (DMSO, $\delta$ ppm, $300 \mathrm{MHz}): 2.15\left(\mathrm{~s}, 3 \mathrm{H},-\mathrm{CH}_{3}\right), 2.22\left(\mathrm{~s}, 3 \mathrm{H},-\mathrm{CH}_{3}\right), 2.50-(\mathrm{br}, 10 \mathrm{H}, \mathrm{N}-$ $\left.\left(\mathrm{CH}_{2}\right)_{5}\right), 2.60$ (br, 4H, $\left.\mathrm{CH}_{3}-\mathrm{N}\left(\mathrm{CH}_{2}\right)_{2}\right), 2.69$ (br, 4H, Ar-N $\left.\left(\mathrm{CH}_{2}\right)_{2}\right), 7.40-7.44$ (m, 7H. ArH), $9.14(\mathrm{~s}, 1 \mathrm{H}, \mathrm{NH}), 10.18(\mathrm{~s}, 1 \mathrm{H}, \mathrm{OH})$

Antioxidant activity

All the synthesized compounds were screened for antioxidant potential in vitro by reducing power activity and hydrogen peroxide-scavenging activity (Table 1 and 2).

Reducing power activity by $\mathrm{FeCl}_{3}$

Reducing power (RP) of synthesized compounds was determined according to the method of Oyaizu $^{13}$. Different aliquots of the test sample and ascorbic acid as standard for comparison at concentration of $50 \mu \mathrm{g} / \mathrm{mL}, 100 \mu \mathrm{g} / \mathrm{mL}, 150 \mu \mathrm{g} / \mathrm{mL}, 200 \mu \mathrm{g} / \mathrm{mL}$ and $250 \mu \mathrm{g} / \mathrm{mL}$ were 
taken in different test tubes. $2.5 \mathrm{~mL}$ Phosphate buffer $(\mathrm{pH} 6.6)$ and $2.5 \mathrm{~mL}$ of $1 \% \mathrm{~K}_{3} \mathrm{Fe}(\mathrm{CN})_{6}$ were added in each test tube. Test solutions were kept for 20 minutes at $50{ }^{\circ} \mathrm{C}$ in water bath. After 20 minutes $2.5 \mathrm{~mL} 10 \%$ trichloro acetic acid was added in each test solution. An aliquot of $2.5 \mathrm{~mL}$ was withdrawn from each test solution and in it $2.5 \mathrm{~mL}$ distilled water and $1.0 \mathrm{~mL} \mathrm{FeCl}_{3}(0.1 \%)$ were added. A blank was also prepared without adding the test compound. Each experiment was carried out in triplicate and mean value was calculated. Finally the antioxidant activity was evaluated by determine the absorbance at $700 \mathrm{~nm}$ after 10 minutes.

\section{Hydrogen peroxide scavenging activity}

The Hydrogen peroxide scavenging activity was determined by the method of Ruch et al., ${ }^{14}$ The synthesized compounds were dissolved in $3.4 \mathrm{~mL}$ of $0.1 \mathrm{M}$ phosphate buffer $(7.4 \mathrm{p} \mathrm{H})$ and mixed with $600 \mu \mathrm{L}$ of $43 \mathrm{mM}$ solution of hydrogen peroxide. The absorbance value at $230 \mathrm{~nm}$ of the test samples were recorded at 10 minutes intervals between 0 to 40 minutes. BHT was used as standard for comparison.

\section{Antibacterial activity}

The antimicrobial activity of all the synthesized Schiff bases were examined against S.aureus, E.coli, K.pneumoniae bacterial strains by measuring the zone of inhibition. The antibacterial activity was performed by Agar well diffusion method ${ }^{15}$ at a concentration level of $250 \mu \mathrm{g} / \mathrm{mL}$ and $500 \mu \mathrm{g} / \mathrm{mL}$ following reported procedure. Streptomycin was used as the standard at the same concentration levels. Nutrient agar was used as the culture media for antibacterial activity and DMF was used as control. The results are shown as bar diagram (Figure 1).

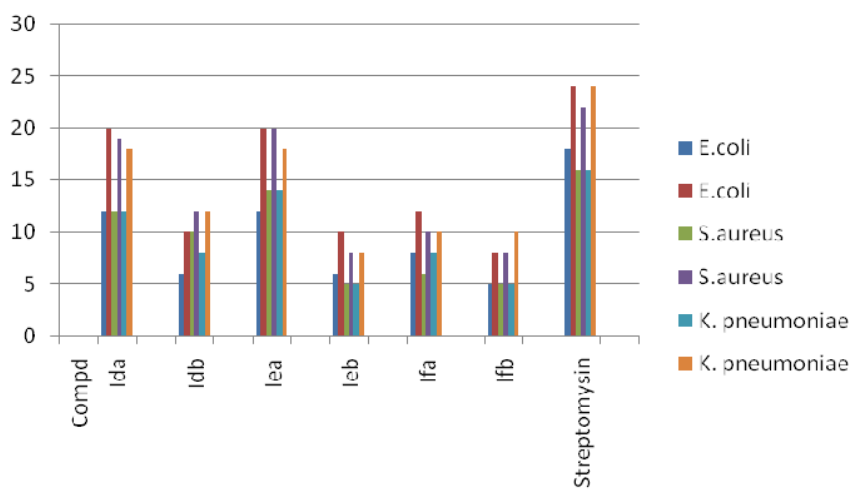

Figure 1. Antibacterial activity of synthesized compounds

Table 1. Antioxidant activity of synthesized compounds by reducing power assay

\begin{tabular}{cccccc}
\hline \multirow{2}{*}{ Compd } & \multicolumn{5}{c}{ \% Inhibition } \\
\cline { 2 - 6 } & $50 \mu \mathrm{g} / \mathrm{mL}$ & $100 \mu \mathrm{g} / \mathrm{mL}$ & $150 \mu \mathrm{g} / \mathrm{mL}$ & $200 \mu \mathrm{g} / \mathrm{mL}$ & $250 \mu \mathrm{g} / \mathrm{mL}$ \\
\hline $\mathbf{I d}_{\mathbf{a}}$ & 10 & 23 & 33 & 45 & 56 \\
$\mathbf{I d}_{\mathbf{b}}$ & 15 & 32 & 48 & 68 & 76 \\
$\mathbf{I e}_{\mathbf{a}}$ & 16 & 38 & 52 & 69 & 78 \\
$\mathbf{I e}_{\mathbf{b}}$ & 9 & 24 & 38 & 66 & 70 \\
$\mathbf{I f}_{\mathbf{a}}$ & 12 & 28 & 42 & 67 & 74 \\
$\mathbf{I f}_{\mathbf{b}}$ & 10 & 25 & 30 & 40 & 52 \\
Ascorbic acid & 45 & 60 & 68 & 78 & 87 \\
\hline
\end{tabular}


Table 2. Antioxidant activity of synthesized compounds by Hydrogen peroxide scavenging activity

\begin{tabular}{cccccc}
\hline \multirow{2}{*}{ Compd. } & \multicolumn{5}{c}{ \% Scavenging of $\mathrm{H}_{2} \mathrm{O}_{2}$} \\
\cline { 2 - 6 } & 0 & 10 minutes & 20 minutes & 30 minutes & 40 minutes \\
\hline $\mathbf{I d}_{\mathbf{a}}$ & 85 & 79 & 74 & 69 & 65 \\
$\mathbf{I d}_{\mathbf{b}}$ & 69 & 66 & 62 & 57 & 54 \\
$\mathbf{I e}_{\mathbf{a}}$ & 66 & 63 & 59 & 55 & 52 \\
$\mathbf{I}_{\mathbf{b}}$ & 78 & 74 & 71 & 67 & 62 \\
$\mathbf{I f}_{\mathbf{a}}$ & 72 & 68 & 64 & 60 & 55 \\
$\mathbf{I f}_{\mathbf{b}}$ & 74 & 71 & 66 & 58 & 53 \\
$\mathrm{BHT}$ & 94 & 83 & 75 & 72 & 68 \\
\hline
\end{tabular}

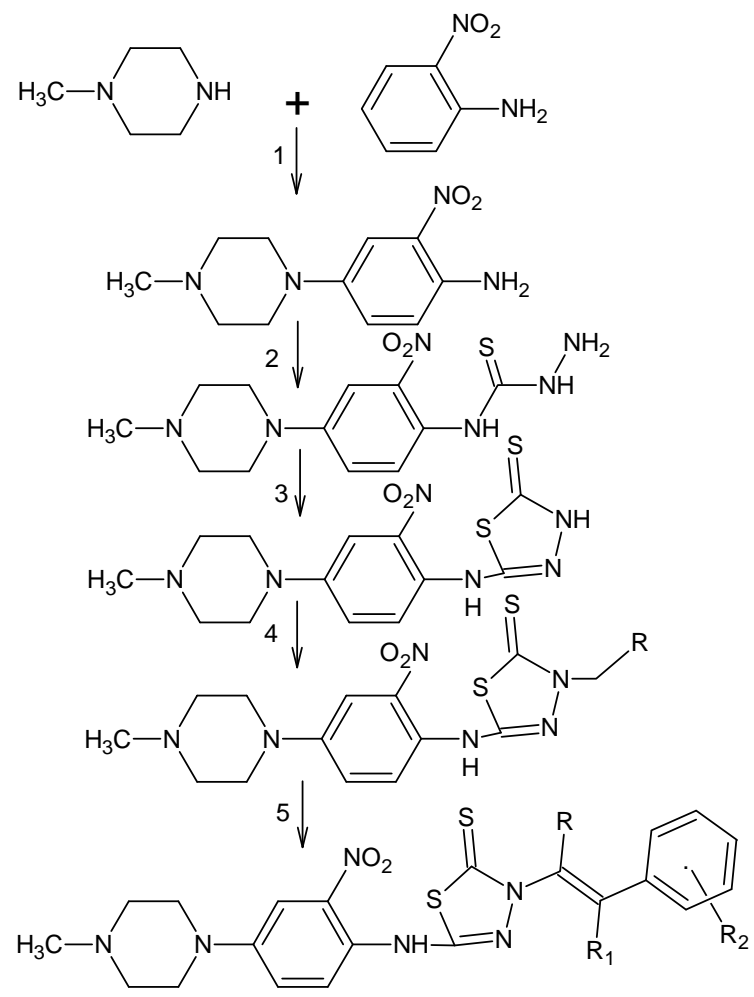

1. Benzene, 2. $\mathrm{CS}_{2}, \mathrm{NH}_{2}-\mathrm{NH}_{2}$ and $\mathrm{ClCH}_{2} \mathrm{COONa}$ in $\mathrm{NH}_{3}, 3 . \mathrm{CS}_{2}$ in DMF, 4. $\mathrm{HCHO}$ and Substituted amines in ethanol, 5. Carbonyl compound and anhydrous sodium acetate in acetic acid, $\mathrm{R}=$ anisidino/morpholino/piperidino, $\mathrm{R}_{1}=\mathrm{H}, \mathrm{CH}_{3}, \mathrm{R}_{2}=$ 4-hydoxy,3-methoxy-benzilidene, 4-hydroxybenzilidene

Scheme 1. Systematic reaction of synthesized compounds

\section{Results and Discussion}

Antimicrobial activity

Four derivatives namely $\mathbf{I d}_{\mathbf{b}}, \mathbf{I} \mathbf{e}_{\mathbf{b}}, \mathbf{I} \mathbf{f}_{\mathbf{a}}$ and $\mathbf{I f}_{\mathbf{b}}$ have shown moderate inhibition against all the three tested strains, while two derivatives $\mathbf{I d}_{\mathbf{a}}$ and $\mathbf{I}_{\mathbf{a}}$ being highly active $(20-24 \mathrm{~mm})$ comparable to Streptomycin against all the three strains as shown by high bars (Figure 1). 


\section{Antioxidant activity}

The antioxidant activity in terms of reducing power shows that as the concentration of the test compounds increase there is increase in the reducing power of these derivatives. Among the six derivatives synthesized, maximum reducing potential is observed in compound $\mathbf{I e}_{\mathbf{a}}$. However less activity is observed compared to ascorbic acid.

The antioxidant activity in terms of hydrogen peroxide scavenging potential shows that oxidation power of the synthesized compounds decreases with increase in time. Hydrogen peroxide scavenging values of $85 \%, 79 \%, 74 \%, 69 \%$ and $65 \%$ was observed in a span of 0 to 40 minutes for compound $\mathbf{I d}_{\mathbf{a}}$, which was maximum among the six tested compounds. It is also know that greater the oxidation power lesser is the reducing capacity. This phenomenon is evident and observed in compound $\mathbf{I d}_{\mathbf{a}}$ where the oxidation potential is highest while the reducing power is least.

\section{Conclusion}

Microwave assisted synthesis can be used to reduce the time and increase the yield of reaction. The bioactivity results proved that synthesized Schiff bases can be used for the treatment of diseases caused by microbes and compounds may be potential in exploring new antioxidant lead drugs.

\section{Acknowledgement}

I would like to thank my Ph.D. research guide Professor Mrs. Pramilla Sah whose able guidance has helped me to complete my project successfully. The incredible encouragement, co-operation and perfect guidance of my guide are my greatest privilege. His constant encouragement and eminent guidance helped me to complete the task to best of my ability. I would like to extend my deep and sincere thanks to Mr. M.Y. Multani, Principal Govt. College Merta City for providing infrastructure facilities for smooth conducting the research work. I am sincerely thankful to Dr. Kapil Geglot, Head Department of Chemistry, Lachoo Mamorial College of Science and technology, Jodhpur for his support during antioxidant activity. I want to extend my sincere thanks to Dr Neetu Bharatiya, Head Department of Chemistry, Government College Merta City for her kind co-operation. I am also very thankful to Head Department of Botany, Jai Narayan Vyas University for providing facilities for antibacterial activity. I also want to thank UGC, New Delhi for their financial assistance under minor research project.

\section{References}

1. Karale B K, Tarkate S J, Salve S P, Zaware B H and Jadhav S S, Indian J Chem., 2014, 53B, 339-344.

2. Sah P, Bidawat P, Seth M and Gharu C P, Arab J Chem., 2014, 7(2), 181-187; DOI:10.1016/j.arabjc.2010.10.023

3. $\quad$ Sah P and Gharu C P, J Cur Pharm Res., 2012, 9(1), 44-48.

4. Talath S and Gadad A K, Eur J Med Chem., 2006, 41(8), 918-924, DOI:10.1016/j.ejmech.2006.03.027

5. Foroumadi A, Rineh A, Emami S, Siavoshi F, Massarrat S, Safari F, Saeed R, Mehraban F, Ensieh L and Abbas S, Bioorg Med Chem Lett., 2008, 18(11), 33153320; DOI:10.1016/j.bmcl.2008.04.033

6. Joshi N K, Kundariya D S and Parmar J M, Int J Chem Tech Res., 2012, 4(4), 1503-1508.

7. Savaliya M D, Dobariya J G, Kathiriya P J and Purohit D M., Indian J Chem., 2011, 20(3), 249-252. 
8. $\quad$ Sah P and Gharu C P, Paripex Indian J Res., 2013, 2(3), 15-17.

9. Shaaban M R, Saleh T S, Mayhoub A S, Mansour A. and Faraq A M, Bioorg Med Chem., 2008, 16(12), 6344-6352; DOI:10.1016/j.bmc.2008.05.011

10. Rajasekaran S, Rao G K, Pai P N S, Ahmed Q and Jasmine A, Indian J Hetrocycl Chem., 2009, 19, 191-192.

11. Hossain M M, Kumar S S and Foysal A Bangladesh Med Res Counc Bull., 2009, 35(2), 49-52; DOI:10.3329/bmrcb.v35i2.2564

12. Vijayabhasker K, Vasu K K and Patel P, Indian J Heterocycl Chem., 2013, 23, 47-50.

13. Oyaizu M, Jpn J Nut., 1986, 44, 307-315.

14. Ruch R T, Cheng S J and Klaunig J E, Meth Enzym., 1984, 105, 198-209.

15. Barry A L, Joyce L J, Adams A P and Benner E J, Amer J Clin Pathol., 1973, 45(4), 493-496. 